\title{
Analysis of the Metallic Phase of Two-Dimensional Holes in SiGe in Terms of Temperature Dependent Screening
}

\author{
V. Senz ${ }^{1}$, T. Ihn ${ }^{1}$, T. Heinzel ${ }^{1}$, K. Ensslin ${ }^{1}$, G. Dehlinger ${ }^{2}$, D. Grützmacher ${ }^{2}$, U. Gennser ${ }^{2}$ \\ 1 Laboratory of Solid State Physics, ETH Zürich, CH-8093 Zürich, ${ }^{2}$ Paul Scherrer Institut, CH-5232 Villigen PSI
}

(November 20, 2018)

\begin{abstract}
We find that temperature dependent screening can quantitatively explain the metallic behaviour of the resistivity on the metallic side of the so-called metal-insulator transition in p-SiGe. Interference and interaction effects exhibit the usual insulating behaviour which is expected to overpower the metallic background at sufficiently low temperatures. We find empirically that the concept of a Fermi-liquid describes our data in spite of the large $r_{s} \approx 8$.
\end{abstract}

Conductivity measurements give experimental access to the nature of the electronic ground state of disordered conductors. At zero temperature $(T=0)$ extended wave functions at the Fermi-level result in metallic behaviour (zero or finite resistivity $\rho$ ), while localised wavefunctions lead to insulating properties (infinite resistivity) [1]. Since in experiments the absolute zero is not accessible, the criterion $d \rho / d T>0(d \rho / d T<0)$ measured at the lowest accessible temperatures is thought to indicate a metallic (insulating) ground state.

In two-dimensional (2D) systems it was suggested theoretically [2] and supported experimentally [3, [4] that the ground state at zero temperature is of insulating nature. This belief has been challenged by the interpretation of experiments on high mobility Si-MOSFETs [5]. Later on, experiments on GaAs hole [6 8] and electron gases [9], AlAs electron gases and SiGe hole gases [10 12] were shown to exhibit similar features as observed in the SiMOSFET systems [13]. As a consequence, the existence of a metal-insulator transition (MIT) in 2D-systems has been controversially discussed [14].

In this paper we discuss the metallic behaviour observed in our p-SiGe quantum wells [11]. Interference corrections to the conductivity are extracted from weak localisation studies at low magnetic fields and interaction corrections to the conductivity are obtained from the temperature dependence of the Hall resistivity, similar to Ref. [7]. In addition, following the suggestion in Ref. [15], we compare the remaining (Drude) part of the conductivity with the theory for temperature dependent screening suitable for our system [16]. We arrive at the following conclusions: (I) In the metallic regime the 2Dhole gas in our SiGe samples behaves like an ordinary Fermi liquid and exhibits localising interference and interaction corrections to the conductivity which can be described by conventional theory. (II) The metallic temperature dependence of the resistivity can be described by the theory of temperature dependent screening entering the Drude part of the resistivity.

Our samples were grown by MBE (molecular beam epitaxy). They comprise a $20 \mathrm{~nm} \mathrm{Si}_{0.85} \mathrm{Ge}_{0.15}$ quantum well where the two-dimensional hole gas is formed, sandwiched between undoped Si layers. The structures are remotely doped with boron at a distance of $15 \mathrm{~nm}$ above the quantum well and gated with a Ti/Al Schottky gate. For more details see Ref. [11]. Measurements were carried out at temperatures between $180 \mathrm{mK}$ and $15 \mathrm{~K}$ using four-terminal AC and DC techniques.

The SiGe quantum well is compressively strained normal to the growth direction, which leads, together with the confinement, to a splitting between heavy-hole $(\mathrm{HH})$ and light-hole $(\mathrm{LH})$ bands of the order of $25 \mathrm{meV}$ [17. The $2 \mathrm{D}$ hole gas resides in the lowest $\mathrm{HH}$ subband with effective mass $m^{\star}=0.25 m_{0}$ as determined from Shubnikov-de Haas oscillations. The hole density in ungated areas of the device was $4.3 \times 10^{11} \mathrm{~cm}^{-2}$. With the gate the hole density could be tuned between $1.1-2.6 \times 10^{11} \mathrm{~cm}^{-2}$, i.e. the Fermi energy was below $2 \mathrm{meV}$. The hole mobility in these structures increases with carrier concentration from $1000 \mathrm{~cm}^{2} / \mathrm{Vs}$ at the lowest to $7800 \mathrm{~cm}^{2} / \mathrm{Vs}$ at the highest density similar to other p-SiGe structures [18]. The ratio of the Drude scattering time $\tau_{e}$ and the quantum lifetime $\tau_{q}$ extracted from Shubnikov-de Haas oscillations is close to one indicating that transport is dominated by short range scattering potentials. It was confirmed in other studies that large angle scattering at interface charges dominates the mobility in such structures [17,18.

Recently Coleridge et al. [10 and we 11, 12 have shown that p-SiGe exhibits the characteristic features of the MIT. On increasing the hole density $d \rho / d T$ changes sign [11]. At the critical density $p_{c}$, we found $r_{s}=$ $1 / a_{B}^{\star} \sqrt{\pi p} \approx 8$, where $a_{B}^{\star}$ is the effective Bohr radius. The critical resistance was $\rho_{c} \approx h / e^{2}$. The $r_{s}$ value is a measure of the importance of interaction effects and $\rho$ is a measure of the degree of disorder in the system. Like in other systems temperature and electric field scaling could be performed 11] allowing the interpretation in terms of a quantum phase transition. We have found in Ref. [12] that in the metallic regime weak localisation (WL) reduces the metallic behaviour without destroying it, whereas localising interference corrections dominate the zero field resistivity in the insulating regime.

In the following we analyse magnetotransport data in the metallic regime by first extracting the contributions of WL and interaction corrections to the temperature 
dependence of the conductivity; then the effect of temperature dependent screening is quantitatively discussed.

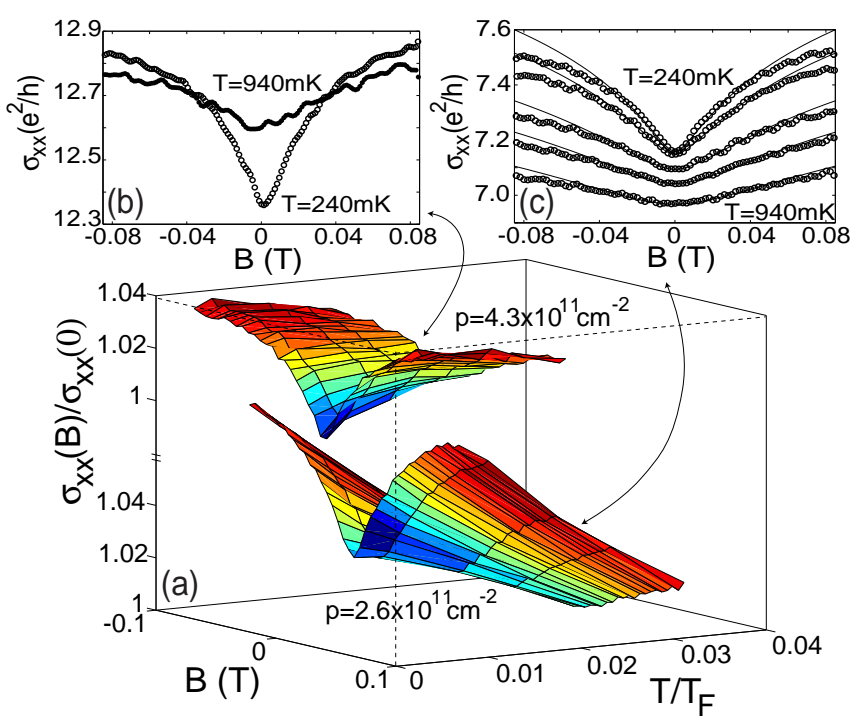

FIG. 1. (a) Temperature and magnetic field dependence of the conductivity for densities $p=2.6 \times 10^{11} \mathrm{~cm}^{-2}$ (lower surface) and $p=4.3 \times 10^{11} \mathrm{~cm}^{-2}$ (upper surface). The upper surface shows reentrant insulating behaviour at lower temperatures. (b) Magnetoconductance for the higher density at the two extreme temperatures. (c) Magnetoconductance for the lower density at various temperatures.

In Fig. 1 a we show magnetoconductivities measured at the highest densities $p=2.6 \times 10^{11} \mathrm{~cm}^{-2}$ (achieved under the gated part of the sample) and $p=4.3 \times 10^{11}$ $\mathrm{cm}^{-2}$ (ungated part of the sample) for various temperatures. Fig. 1 b presents the magnetoconductivity curves of the higher density for the lowest and highest temperature. Fig. 11c shows magnetoconductivity curves of the lower density together with theoretical fits (solid lines) to be discussed later in this paper. It can be seen in Fig. 11c that the temperature dependence of the conductivity minimum around zero magnetic field, which we attribute to the WL corrections, is overpowered by the much stronger temperature dependence of the background conductivity. We therefore analyse the zero-field conductivity $\sigma(T)$ in the spirit of Ref. 19.

$$
\sigma(T)=\sigma_{D}(T)+\delta \sigma_{W L}(T)+\delta \sigma_{I}(T),
$$

where $\sigma_{D}(T)$ is the Drude conductivity, $\delta \sigma_{W L}(T)$ and $\delta \sigma_{I}(T)$ are the WL and the interaction contributions, respectively. Strictly speaking, eq. (11) is based on the validity of the Fermi-liquid description and $r_{s} \leq 1$ is required. Nevertheless we empirically apply this concept to our system, since phenomenologically the magnetoconductivity exhibits all the features also found in samples with low $r_{s}$.

In the well established theory of WL 20 spin-orbit relaxation mechanisms like the Elliott-Yafet mechanism, the Dyakanov-Perel mechanism or the Rashba-effect have been taken into account perturbatively [20,21], which is appropriate for conduction band electrons. WL in $2 \mathrm{D}$ p-type systems such as p-GaAs has only recently been studied in detail 22]. In hole gases the valence band is strongly influenced by spin-orbit interaction and strain. The spin relaxation time is of the same order as the momentum relaxation time and can therefore no longer be treated perturbatively [23]. It is therefore not a priori clear that the theories in Refs. 20,21] can be applied to our system.

Here we apply the theory developed in Ref. 23] for ptype $2 \mathrm{D}$ hole gases which takes the valence band structure into account and includes the effect of HH-LH mixing. Fig. 11c shows theoretical curves fitted to the measured conductivity according to

$$
\begin{aligned}
& \delta \sigma_{W L}(B, T)-\delta \sigma_{W L}(0, T)=\frac{e^{2}}{2 \pi^{2} \hbar} \times \\
& \quad\left[f_{2}\left(\frac{B}{B_{\varphi}+B_{\|}}\right)+\frac{1}{2} f_{2}\left(\frac{B}{B_{\varphi}+B_{\perp}}\right)-\frac{1}{2} f_{2}\left(\frac{B}{B_{\varphi}}\right)\right]
\end{aligned}
$$

Here $f_{2}(x)=\ln x+\psi(1 / 2+1 / x), \psi$ is the digamma function and $B_{i}=\hbar /\left(4 D e \tau_{i}\right) \quad(i=\|, \perp, \varphi)$, with the diffusion constant $D$ and the phase-coherence time $\tau_{\varphi}$. The other relaxation times are given in Ref. [23] to be $1 / \tau_{\|}=1 / \tau_{e} \cdot\left(k_{F} a / \pi\right)^{4} I_{\|}$and $1 / \tau_{\perp}=1 / \tau_{e} \cdot\left(k_{F} a / \pi\right)^{6} I_{\perp}$, where $\tau_{e}$ is the transport relaxation time, $a$ is the width of the quantum well and $k_{F}$ is the Fermi wavevector. The quantities $I_{\| / \perp}$ depend on the ratio of the LH and $\mathrm{HH}$ mass and have to be computed numerically. In our samples the holes are effectively confined by a triangular well due to the asymmetric doping and we estimate $k_{F} a / \pi \approx 0.2$. We use $B_{\varphi}, B_{\perp}$ and $B_{\|}$as fitting parameters and find that $B_{\|}, B_{\perp} \ll B_{\varphi}$, i.e. HH-LH mixing seems to be insignificant in our sample. This result is in contrast to the measurements on p-GaAs, where stronger HH-LH mixing leads to appreciable values for $B_{\perp}$ and $B_{\|}$22]. In our case eq. (2) reduces to the result for negligible spin-orbit scattering [20] already used in Ref. [12]. As it was evaluated there the phase breaking rate is $1 / \tau_{\varphi} \propto T$ and it increases with decreasing hole concentration as expected from theory.

Interaction corrections to the conductivity can be extracted from the temperature dependence of the Hallresistance at small magnetic fields [4]. It is predicted that the WL correction does not affect the Hall resistance, while interaction corrections obey [19 $\delta \sigma_{I}=$ $-\sigma \delta R_{H} / 2 R_{H}=e^{2} /\left(2 \pi^{2} \hbar\right)\left(1-3 F^{\star} / 4\right) \ln \left(k T \tau_{e} / \hbar\right)$, where $R_{H}=d \rho_{x y} / d B$ is the Hall constant. Figure 2 shows $\delta \sigma_{I}(T)$ determined in this way for two different densities in the metallic regime. We find that $\delta \sigma_{I}$ is negative and determine $F^{\star}=0.91$ in agreement with Ref. [24]. This means that interaction corrections to the conductivity give another contribution of insulating behaviour to the total conductivity in the metallic phase. This re- 
sult which agrees with Ref. [7] for p-GaAs is of special importance, since interaction corrections have been suggested to lead to metallic behaviour.

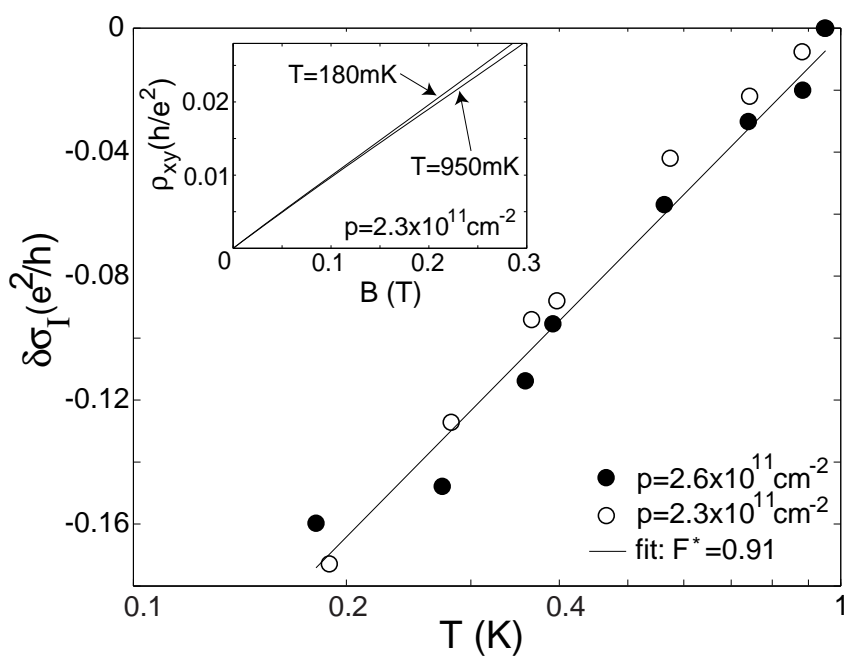

FIG. 2. Interaction corrections $\delta \sigma_{I}(T)$ in the metallic regime. The solid line is a theoretical fit (see text). The inset shows the raw data for the highest and lowest temperature.

Now that we have experimentally determined $\delta \sigma_{W L}(T)$ and $\delta \sigma_{I}(T)$ we are in the position to extract the bare $\sigma_{D}(T)$ according to eq. (11) by subtracting the two corrections from the measured zero-field conductivity (Fig. 3). The obtained Drude conductivity shows a linear metallic temperature dependence which we will address in the following.

The temperature dependent Drude conductivity is

$$
\sigma_{D}(T)=\frac{p e^{2} \tau_{e}(T)}{m^{\star}}
$$

where $p$ is the sheet density of the holes and the average Drude scattering time $\tau_{e}(T)$ has to be calculated according to

$$
\tau_{e}(T)=\frac{\int d E E \tau_{e}(E, T)(-d f / d E)}{\int d E E(-d f / d E)}
$$

with the Fermi distribution function $f(E, T)$ and the energy dependent scattering rate

$$
\frac{\hbar}{\tau_{e}(E, T)}=2 \pi N_{i} \int \frac{d^{2} k^{\prime}}{(2 \pi)^{2}}\left|\frac{V(q)}{\varepsilon(q, T)}\right|^{2}(1-\cos \theta) \delta\left(E_{k}-E_{k^{\prime}}\right)
$$

Here $N_{i}$ is the density of ionized impurities, $V(q)$ is the matrix element for scattering by a wavevector $q=$ $k_{F} \sqrt{2(1-\cos \theta)}$ and $\varepsilon(q, T)$ is Lindhard's dielectric function. For dominant large angle scattering (i.e. scattering for $q \approx 2 k_{F}$ ) it has been shown that 16]

$$
\sigma_{D}(T)=\sigma_{D}(0)\left[1-C(p) \frac{T}{T_{F}}\right]+\mathcal{O}\left[\left(\frac{T}{T_{F}}\right)^{3 / 2}\right]
$$

The linear term stems from the temperature dependence of the dielectric function $\varepsilon(q, T)$, which becomes particularly important for large angle scattering. It is a direct consequence of electron-electron interactions causing a temperature dependent polarizability of the 2D hole gas. Theory predicts values for the constant $C(p)$ which depend on the scattering mechanism and on the hole density $p$. If we apply eq. (3) to $\sigma_{D}(T)$ in Fig. 3 we determine $C=3.1$ in reasonable agreement with a predicted value of $C \approx 2.8$ for charged interface impurity scattering at low $p$. Similar agreement has been found for all densities in the metallic regime. This demonstrates that temperature dependent screening can indeed explain the metallic temperature dependence of the resistivity in our p-SiGe system without invoking a novel metallic phase. It also implies that at sufficiently low temperatures insulating behaviour with $d \rho / d T<0$ is expected to be recovered. Similar results in p-SiGe were obtained for much higher electron densities in Ref. [24].

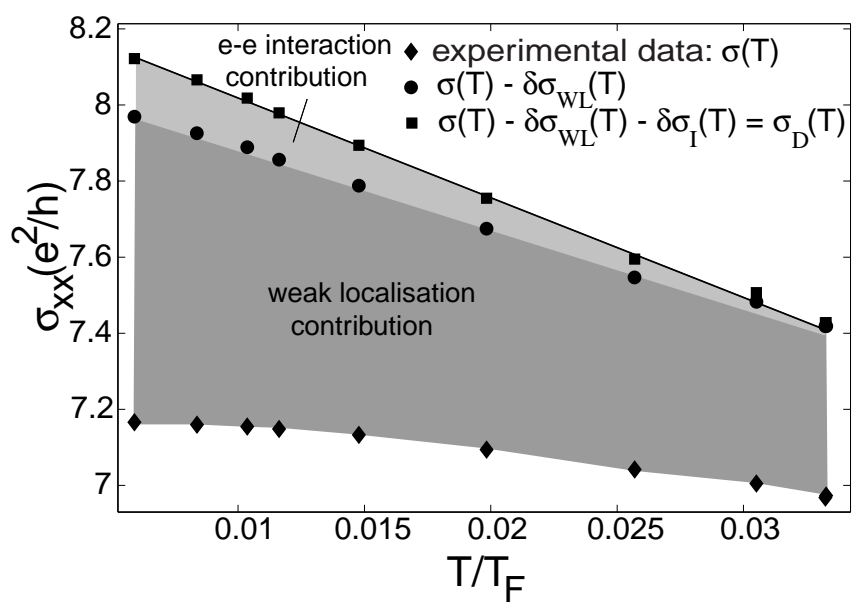

FIG. 3. Zero-field contributions to the conductivity at $p=2.6 \times 10^{11} \mathrm{~cm}^{-2}$. From the measured curve $\sigma(T)(\checkmark)$ we first subtract $\delta \sigma_{W L}(T)$ arriving at the curve marked • and then $\delta \sigma_{I}(T)$ which leaves us with the bare $\sigma_{D}(T)(\boldsymbol{\square})$.

For higher carrier densities $p$ we expect $\sigma_{D}(T)$ to exhibit a weaker temperature dependence, since $C(p)$ decreases [16]. In Fig. 11a we therefore show the magnetic field and the temperature dependence measured at two densities. The lower surface shows the curves measured at $p=2.6 \times 10^{11} \mathrm{~cm}^{-2}$. At large $|B|$ the metallic temperature dependence can be seen. At $B=0$ the $\mathrm{WL}$ peak tends to counteract this metallic behaviour without overpowering it in the range of temperatures shown. The upper surface was measured at a density of $4.3 \times 10^{11}$ $\mathrm{cm}^{-2}$ on an ungated part of the same sample. The temperature dependence at large $|B|$ is weaker than for the other surface $(C=0.8)$, in agreement with temperature dependent screening. At $B=0$ however, the WL correction is strong enough to restore the insulating temper- 
ature dependence of the conductivity. Such a reentrant insulating behaviour is consistent with the observations made in Ref. 25.

In the following, we address the range of validity of our analysis. The theories of linear screening, WL and interaction corrections are expected to hold as long as the disorder in the system is low enough, i.e. as long as $k_{F} l>1$. Therefore, our analysis is limited to the metallic regime. Non-linear screening models have to be invoked at the MIT, where our system undergoes the transition from WL $\left(k_{F} l>1\right)$ to strong localisation $\left(k_{F} l<1\right)$. We have empirically applied concepts developed for weakly interacting systems with $r_{s} \leq 1$. This attempt was successful and a Fermi-liquid description of the 2D hole gas appears to be consistent with the experiment, in spite of $r_{s} \approx 8$. The theoretical understanding and interpretation of this finding remains an open issue.

How relevant is this analysis for other systems in which the MIT was observed? Our analysis was simplified by the fact that large angle scattering is dominant in p-SiGe. For this special case, the analytical results of Ref. 16 apply. Candidates for a similar analysis are therefore low-mobility Si-MOSFETS (for which this theory was originally developed) or the n-GaAs system with InAs quantum dots near the 2D electron gas [9]. However, Kravchenko et al. stated in Ref. [5] explicitly that temperature dependent screening can not account for the metallic behaviour in their high-mobility Si-MOSFET samples. Performing a similar analysis on the n-type GaAs samples of Ref. [9] we find that the temperature dependence in the metallic range is much too large compared to the theory used above. For p-type GaAs samples exhibiting the MIT temperature dependent screening was suggested in Refs. [7, 8] as the relevant mechanism. Under the condition of small angle scattering this effect is much weaker than in our case.

In conclusion, we have analysed the magnetoresistance and the Hall resistance in p-SiGe samples in terms of interference and interaction corrections to the conductivity and found the measurements to be consistent with ordinary Fermi-liquid behaviour in spite of $r_{s} \approx 8$. Both corrections tend to localise the system as the temperature is lowered. The temperature dependence of the background Drude-conductivity has been found to depend linearly on temperature. Its behaviour is in good agreement with the theory of temperature dependent screening for systems in which large angle scattering dominates. The analysis applies to densities where the system is in the metallic regime and can not easily be extended to the transition region, where $k_{F} l \approx 1$. Although these observations can not exclude the possibility of a novel metallic ground state in our or in other systems unambiguously, we are inclined to discard this exciting possible interpretation for $\mathrm{p}$-SiGe hole gases on the basis of our experiments and analysis. The theoretical understanding of experi- mental consistency with Fermi-liquid behaviour at these large $r_{s}$-values remains a challenging topic for further research.

We have enjoyed fruitful discussions with V. Dolgopolov and J.L. Pichard. Financial support from ETH Zurich and the Schweizerischer Nationalfonds is gratefully acknowledged.

[1] P.W. Anderson, Phys. Rev. 109, 1492 (1958).

[2] E. Abrahams et al., Phys. Rev. Lett. 42, 673 (1979).

[3] G.J. Dolan, D.D. Osheroff, Phys. Rev. Lett. 43, 72 (1978); N. Giordano, Phys. Rev. B 22, 5635 (1980); D.J. Bishop et al., Phys. Rev. Lett. 44, 1153 (1980) and 46, 360 (1981) and Phys. Rev. B 24, 773 (1982); M.J. Uren et al., J. Phys. C13, L986 (1980) and C14, L395 (1981); Y. Kawaguchi, S. Kawaji, J. Phys. Soc. Jpn. 48, 699 (1980); R.G. Wheeler, Phys. Rev. B 24, 4645 (1981); B.J.F. Lin et al., Phys. Rev. B 29, 927 (1984).

[4] D.A. Poole et al., J. Phys. C14, L995 (1981).

[5] S.V. Kravchenko et al., Phys. Rev. B 50, 8039 (1994).

[6] Y. Hanein et al., Phys. Rev. Lett. 80, 1288 (1998); J. Yoon et al., Phys. Rev. Lett. 82, 1744 (1999).

[7] M.Y. Simmons et al., Phys. Rev. Lett. 84, 2489 (2000).

[8] A.R. Hamilton et al., cond-mat/0003295, unpublished.

[9] E. Ribeiro et al., Phys. Rev. Lett. 82, 996 (1999).

[10] P.T. Coleridge et al., Phys. Rev. B 56, R12764 (1997).

[11] V. Senz et al., Ann. Phys. (Leipzig) 8, SI 237 (1999).

[12] V. Senz et al., Phys. Rev. B 61, R5082 (2000).

[13] S.V. Kravchenko et al., Phys. Rev. B 59, R12740 (1999); S.V. Kravchenko, Braz. J. Phys. 29, 623 (1999).

[14] G. Benenti et al., Phys. Rev. Lett. 831826 (1999); T. Vojta et al., Phys. Rev. Lett. 81, 4212 (1998); J.S. Thakur, D. Neilson, Phys. Rev. B 59, R5280 (1999); B.L. Altshuler, D.L. Maslov, Phys. Rev. Lett. 82, 145 (1999); Y. Meir, Phys. Rev. Lett. 83, 3506 (1999); see also References in [13,12].

[15] S. Das Sarma et al., Phys. Rev. Lett. 83, 164 (1999).

[16] A. Gold et al., Phys. Rev. B 33, 1076 (1986).

[17] C.J. Emeleus et al., J. Appl. Phys. 73, 3852 (1993).

[18] T.E. Whall, Appl. Surf. Sci. 102, 221 (1996).

[19] B.L. Altshuler, A.G. Aronov, Electron-Electron Interaction In Disordered Conductors, editors A.L. Efros and M. Pollak, Elsevier Science Publishers B.V., 1985.

[20] S. Hikami et al., Progr. Theor. Phys. 63, 707 (1980).

[21] B.L. Altshuler et al., Sov. Phys. JETP 54, 411 (1981); S.V. Iordanskii et al., JETP Lett. 60, 206 (1994); F.G. Pikus, G.E. Pikus, Phys. Rev. B 51, 16928 (1995); W. Knap et al., Phys. Rev. B 53, 3912 (1996); T. Hassenkam et al., Phys. Rev. B 55, 9298 (1997).

[22] S. Pedersen et al., Phys. Rev. B 60, 4880 (1999).

[23] N.S. Averkiev et al., JETP 86, 780 (1998).

[24] C.J. Emeleus et al., Phys. Rev. B 47, 10016 (1993).

[25] A.R. Hamilton et al., Phys. Rev. Lett. 82, 1542 (1999); V.M. Pudalov et al., Phys. Rev. B 60, R2154 (1999). 